\section{THE DIFFERENCE IN EXTERNAL RADIATION EXPOSURE DEPENDING ON THE WORKING PROCESS AMONG RADIATION DECONTAMINATION WORKERS IN FUKUSHIMA}

Takeyasu Kakamu*, Tomoo Hidaka, Tomohiro Kumagai, Yusuke Masuishi, Shota Endo, Sei Sato, Tetsuhito Fukushima. Department of Hygiene and Preventive Medicine, Fukushima Medical University, School of Medicine, Fukushima, Japan

\subsection{6/oemed-2018-ICOHabstracts.1196}

Introduction Since the occurrence of the Fukushima Daiichi Nuclear Power Plant Accident, radiation decontamination work has been conducted in Fukushima. In this work, several different working processes are typically performed simultaneously in a single area. However, the difference in external radiation dose rates between processes is unknown. The aim of this study was to compare external radiation exposure of decontamination workers between processes.

Methods The subjects were 130 radiation decontamination workers who worked at the same site from April to December 2016. We obtained the external radiation dose rates from a radiation decontamination company, whose workers wear external radiation dose monitors at all times when working. The monitors are used to calculate the external radiation dose rates every month. We used the data from April to December 2016. We divided the workers into 5 groups according to their working processes; administration, removal, collection, and transportation of contaminated soil, and filling of uncontaminated soil. We compared the total radiation dose per month and total overall dose during the period between the groups.

Result The median (25-75 percentile) external radiation dose rate during the period was $0.82(0.72-0.91) \mathrm{mSv}$. The process with the highest exposure was removal of contaminated surface soil, with a median of $0.96(0.91-0.99) \mathrm{mSv}$. The exposure rates of administration and filling of uncontaminated soil were $0.64(0.57-0.71)$ and $0.71 \quad(0.65-0.77)$ respectively, which were lower than those in the other processes.

Discussion The cumulative radiation exposure rates in a single area differed according to working process. Although the differences in radiation exposure were relatively small, cumulative exposure may increase in the long term. This radiation decontamination work is globally unprecedented and the effect of low-dose radiation exposure is unknown. Continuous monitoring of low-dose radiation, as well as surveying its effects, are necessary.

\section{MATERNAL CUMULATIVE EXPOSURE TO EXTREMELY LOW FREQUENCY ELECTROMAGNETIC FIELDS AND PREGNANCY OUTCOMES IN THE ELFE COHORT}

\footnotetext{
${ }^{1} \mathrm{~L}$ Migault ${ }^{*},{ }^{1} \mathrm{C}$ Piel, ${ }^{1,2} \mathrm{C}$ Carles, ${ }^{1} \mathrm{~F}$ Delva, ${ }^{1} \mathrm{~A}$ Lacourt, ${ }^{3} \mathrm{E}$ Cardis, ${ }^{4} \mathrm{C}$ Zaros, ${ }^{5} \mathrm{R}$ de Seze, 1,21 Baldi, ${ }^{1} \mathrm{G}$ Bouvier. ${ }^{1}$ University of Bordeaux, Inserm UMR 1219 EPICENE Team, Bordeaux Population Health Research Centre, Bordeaux, France; ${ }^{2}$ Bordeaux University Hospital, Service de Médecine du Travail et pathologie professionnelle, Bordeaux, France; ${ }^{3}$ ISGlobal, Centre for Research in Environmental Epidemiology, Universitat Pompeu Fabra Barcelona, Spain; ${ }^{4}$ Joint research unit Elfe, Ined-Inserm-EFS, France; ${ }^{5}$ TOXI PERITOX UMI-01 unit, INERIS, Verneuil en Halatte, UPJV, Amiens, France
}

\subsection{6/oemed-2018-ICOHabstracts.1197}

Objectives To study the relations between maternal cumulative exposure to extremely low frequency electromagnetic fields (ELF MF) and the risk of moderate prematurity and small for gestational age within the birth cohort Elfe.
Methods The Elfe study included 18,329 infants born at 33 weeks of amenorrhea or more in France in 2011 and was designed to follow children until 20 years of age. Gestational age and anthropometric data at birth was collected in medical records and small for gestational age was defined according to a French customised growth standard. During interviews, mothers were asked to report their job status during pregnancy. If employed, their occupation was coded according to the ISCO-88 classification and the date on which they stopped their work was recorded. Cumulative exposure to ELF MF during pregnancy was assessed, for both mothers who worked and those who didn't work during pregnancy, using a recently updated job-exposure matrix (JEM). Cumulative exposure was considered as a categorical variable $(<17.5,17.5-23.8,23.8$ $36.2,36.2-61.6$ or $\geq 61.6 \mu$ T-days), as a binary variable (<44.1 and $\geq 44.1 \mu \mathrm{T}$-days) and as a continuous variable. Associations were analysed by logistic regression, adjusting for mother's lifestyle factors, sociodemographic characteristics and some mother's medical history during and before pregnancy. Analyses were restricted to single births and to complete values for the pregnancy outcomes $(n=16,733)$.

Results Cumulative exposure was obtained for $96.0 \%$ of the mothers. Among them, 37.5\% were classified in the 23.8-36.2 $\mu \mathrm{T}$-days category, but high exposures were rare: $1.3 \%$ in the $\geq 61.6 \mu \mathrm{T}$-days category and $5.5 \%$ in the $\geq 44.1 \mu \mathrm{T}$-days category. No significant association was observed between maternal cumulative exposure and moderate prematurity and small for gestational age.

Conclusion This large population-based study does not suggest that maternal exposure to ELF MF during pregnancy is highly associated with risk of moderate prematurity or small for gestational age.

\section{THE ROLE OF NEAR INFRARED WITH REGARD TO POTENTIAL LONG-TERM ADVERSE EFFECTS IN OUTDOOR WORKERS EXPOSED TO SOLAR RADIATION}

C Grandi*, A Militello, M Borra. INAIL - Dept. of Occupational and Environmental Medicine, Epidemiology and Hygiene, Monte Porzio Catone (RM), Italy

\subsection{6/oemed-2018-ICOHabstracts. 1198}

Introduction The near infrared (IRA, 780-1.400 nm) represents a primary component of the solar spectrum, accounting for about $30 \%$ of the total solar energy reaching the soil. IRA is highly penetrating, being able to reach the subcutaneous tissues. Moreover, significant amounts of IRA may reach retinal tissue. Outdoor workers are occupationally exposed to solar radiation. However, until now the attention was primarily focused on the acute and long-term adverse effects (especially skin cancer) involving both skin and eyes due to UVB and UVA bands.

Methods A literature search was conducted to assess the current knowledge about the biological effects of IRA, analysing implications in terms of occurrence of long term adverse effects in people working outdoor.

Results Experimental studies show that IRA may affect gene expression profile and the redox balance of the cell, especially fibroblasts, causing a direct or an indirect increase in ROS production, primarily interfering with mitochondrial activity. Until now there is no epidemiological evidence regarding long-term adverse effects due to solar IRA, by alone or in combination with other bands of solar radiation. 